\title{
ELECTRONIC STRUCTURE OF $f$-ELEMENT SYSTEMS IN THE UV AND VUV ENERGY RANGE
}

\author{
J.C. Krupa, I. Gérard, A. MaYolet \\ Institut de Physique Nucléaire, CNRS-IN2P3, 91406 Orsay Cedex, France \\ AND P. MARTIN
}

LURE Bât. 209 C, 91405 Orsay Cedex, France

The main interest of this work is focused on the localization in energy of the different photon absorption mechanisms occurring in fluoride systems doped with rare earth ions by recording the visible luminescence excited by UV and VUV photons between 5 to $25 \mathrm{eV}$. Low temperature experiments have provided opportunity to measure in good conditions the charge transfer and the excited $4 f^{n-1} 5 d$ configuration transitions in the $\mathrm{LaF}_{3}: \mathrm{Ln}^{3+}, \mathrm{O}^{2-}$ systems ( $\mathrm{Ln}=\mathrm{Eu}, \mathrm{Dy}, \mathrm{Er})$.

PACS numbers: 78.55.Fv

\section{Introduction}

From the point of view of luminescence applications, large band gap materials such as fluoride compounds are suitable matrices into which a small concentration of foreign ions called activators can be incorporated. To investigate the role of such activators in the luminescence process, knowledge of their optical properties is necessary for an improved understanding of the overall system.

Most of inorganic luminescent systems contain dopant ions whose excited states are not densely distributed between the valence and the conduction bands. The clearest illustration is provided by ionic solids doped with elements from the lanthanide series which may have or not, only a very small overlap with the other valence orbitals. Optical excitation in these systems is then expected to take place either in the host where rather delocalized electrons are involved and promoted from filled to empty bands (in the energy band description), or in the rare earth center itself where transitions between quasi-atomic states lead to absorption and emission.

Host sensitized luminescence phenomena suggest a strong coupling between the host energy levels and the impurity states. For the investigation of the energy transfer process to the center of luminescence, excitation spectrum of the visible emission becomes the adapted technique which determines, in addition, the energy 
of the absorption bands within the incident photon energy range. Mechanisms that do not result in transfer of energy to the radiatively decaying level (or are of very low quantum yield) are not detected, although by direct absorption measurements they might be observed to give rise to intense absorption.

The present work will be centered on visible luminescence excitation spectra of lanthanide ions, $\mathrm{Ln}^{3+}$, doped into fluoride compounds containing more or less oxygen ions. Visible luminescence is induced by high energy incident photons, from approximately 5 to $25 \mathrm{eV}$, using the synchrotron radiation. With the availability of such a tunable electromagnetic radiation source, many of the experimental difficulties in probing vacuum ultraviolet (VUV) spectra of solids have been now overcome.

In the VUV energy domain, the optical properties of solids such as fluorides are primarily determined by the electronic structure of bands due to the filled orbitals of fluorine ligands and empty orbitals of the metallic cation. When rare earth ions are introduced into the host lattice, the excitation spectra of their visible fluorescence give information on the electronic structure of the host as well as on the energy levels of the metallic impurity whose structure is more or less distinguishable from the first one. The host deep-lying level sensitization is due to valence band to conduction band transitions, exciton formation, core excitation or multiple excitation phenomena. In contrast, the direct excitation of the luminescent rare earth center is due to excitation of rather localized $f$ electrons involving atomic-type interconfiguration transitions and/or molecular orbital-type transitions when an electron is transferred from the anionic environment to the rare earth impurity cation.

Observed spectral features result in more or less efficient transfer of excitation energy toward the $4 f^{q}$ ground state configuration levels of the doping rare earth ions. Then, temperature dependent experiments should provide information on the energy relaxation in the condensed matter including the dynamics of the excited states.

The main area of applied physics that is based on these studies is dealing with the development of new phosphors which can be excited in the VUV. These phosphors are used in a new generation of lamps and in gas plasma discharge devices for flat colour screens.

\section{Technical procedures}

Excitation spectra were recorded using the synchrotron radiation from the positron storage ring SUPERACO at the Laboratoire d'Utilisation du Rayonnement Electromagnétique (LURE) at Orsay. The synchrotron radiation light was dispersed through a three meters computer driven monochromator maintained at $10^{-9}$ torr and equipped with a $300 \mathrm{gr} . \mathrm{mm}^{-1}$ grating blazed at $63 \mathrm{~nm}$. The dispersion was $1 \mathrm{~nm}$ per $\mathrm{mm}$ and the beam dimension at the sample approximately $3 \mathrm{~mm}^{2}$. The photoelectron current produced on a gold grid bombarded by $20.7 \mathrm{eV}$ $(60 \mathrm{~nm})$ photons with a cross-section yield of 0.12 was used to determine the VUV incident photon intensity and its temporal variation.

The visible emitted light from the samples was collected by a quartz rod 
and guided to Hamamatsu R928 photomultiplier window through appropriate filters. The output signal was converted into frequency in order to be stored in the computer memory vs. excitation wavelength in the 50 to $350 \mathrm{~nm}$ range.

The sample response was calibrated with the excitation spectra of sodium salicylate recorded in the same experimental conditions before and after the sample run. Sodium salicylate emission is centered at $443 \mathrm{~nm}$ and its quantum efficiency was found to be constant in the energy range investigated [1].

$\mathrm{LaF}_{3}: \mathrm{Ln}^{3+}$ powdered samples were obtained by the high temperature diffusion technique. Mixtures of commercially available high purity $\mathrm{LaF}_{3}$ material doped with $1 \%$ in weight of rare earth fluorides such as $\mathrm{EuF}_{3}, \mathrm{DyF}_{3}, \mathrm{ErF}_{3}$ or $\mathrm{TbF}_{3}$ were heated directly up to $1100^{\circ} \mathrm{C}$ in a platinum crucible contained in a quartz tube under argon gas circulation. This procedure permits a small amount of oxygen contamination due to $\mathrm{H}_{2} \mathrm{O}$ hydratation molecules which plays an important role in the visible emission mechanisms occurring in some of the investigated compounds [2]. The resulting luminescent polycrystalline compounds were pressed into cylindrical depressions set in a copper sample holder which can be cooled up to approximately $10 \mathrm{~K}$ by liquid helium circulation.

\section{Results and discussion}

It is important to point out that the relative intensity of features recorded on excitation spectra of the visible luminescence reflects not only the absorption cross-section but both absorption and energy transfer efficiencies. In fact, the
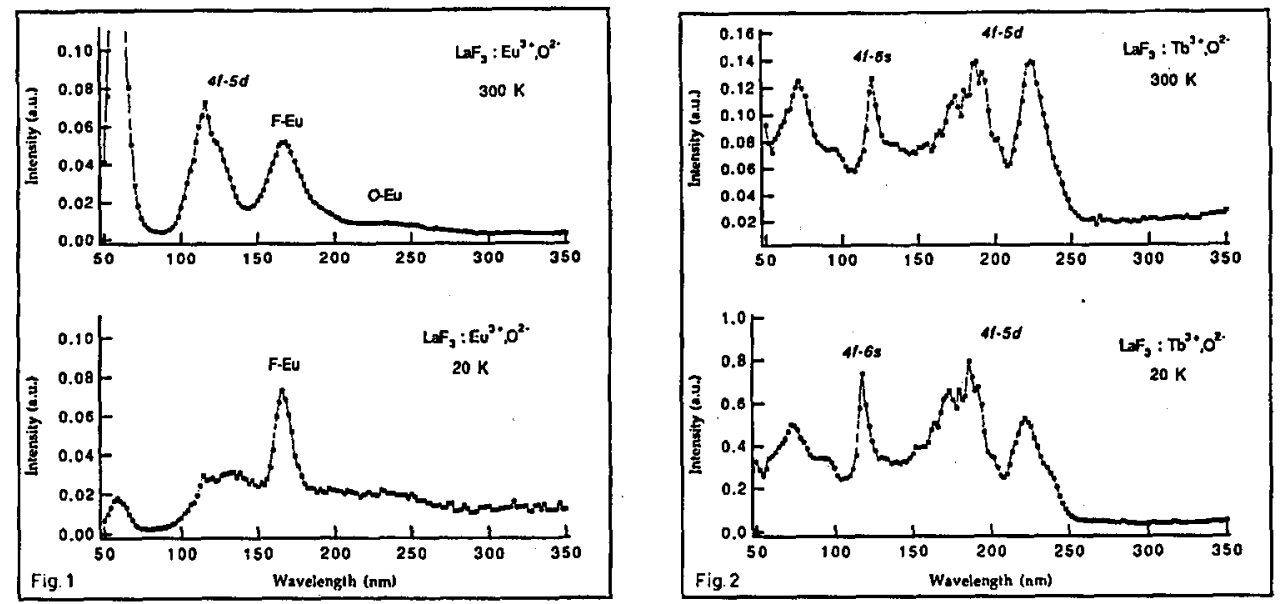

Fig. 1. Excitation spectra of $\mathrm{LaF}_{3}:\left(\mathrm{Eu}^{3+}, \mathrm{O}^{2-}\right)$ in the $\mathrm{UV}$ and VUV range at room and low temperature.

Fig. 2. Excitation spectra of $\mathrm{LaF}_{3}:\left(\mathrm{Tb}^{3+}, \mathrm{O}^{2-}\right)$ in the $\mathrm{UV}$ and $\mathrm{VUV}$ range at room and low temperature. 

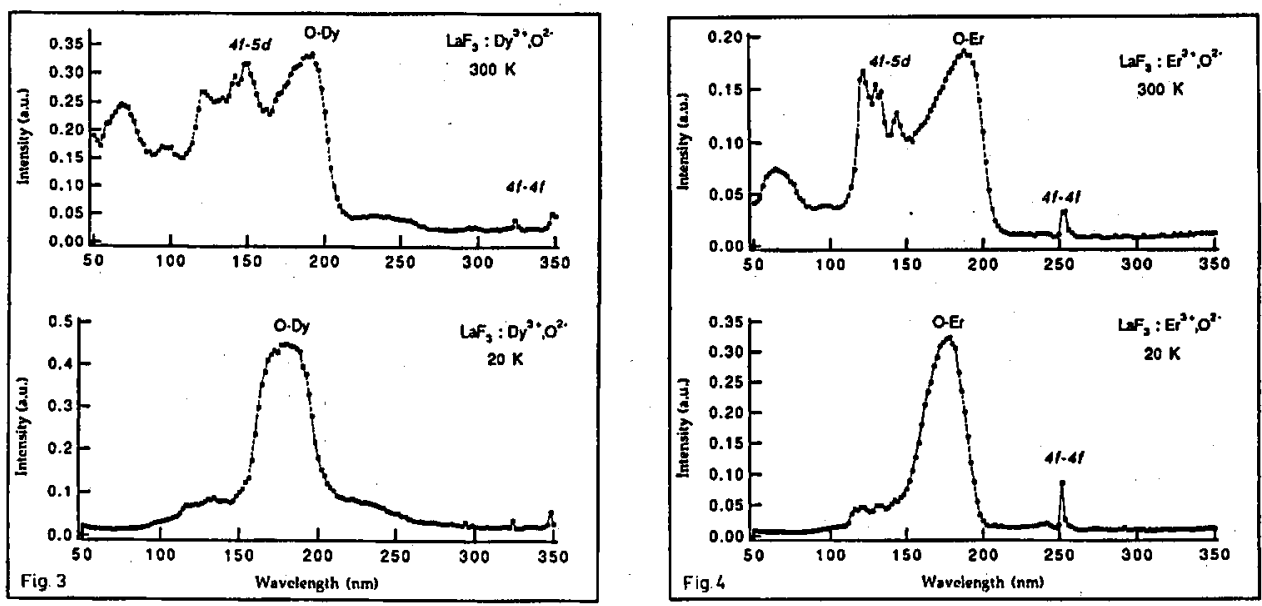

Fig. 3. Excitation spectra of $\mathrm{LaF}_{3}:\left(\mathrm{Dy}^{3+}, \mathrm{O}^{2-}\right)$ in the UV and VUV range at room and low temperature.

Fig. 4. Excitation spectra of $\mathrm{LaF}_{3}:\left(\mathrm{Er}^{3+}, \mathrm{O}^{2-}\right)$ in the UV and VUV range at room and low temperature.

energy transfer process to the radiatively decaying level of the rare earth impurity is the main factor governing the visible luminescence intensity. In the energy range investigated, different energy relaxation mechanisms involving nonradiative and VUV/UV radiative transitions can be reasonably considered. They are competing and their temperature dependencies on the visible emission rate are of interest to understand the luminescence process. For this reason, the excitation spectra of the visible luminescence of $\mathrm{LaF}_{3}$ host matrices doped with $\mathrm{Eu}^{3+}, \mathrm{Dy}^{3+}, \mathrm{Er}^{3+}$, $\mathrm{Tb}^{3+}$ and a small amount of oxygen ions, were recorded at both: room and low temperature (Figs. 1, 2, 3 and 4).

\subsection{Intra-configuralion transitions}

In the high energy region, a relatively strong peak appears at room temperature. The plot of the energy of its maximum, versus the number of $f$ electrons in the ground configuration (Fig. 5), follows the variation of the binding energy of $4 f$ electrons in the respective rare earth fluorides [3]. Then, this absorption peak which is correlated to the nature of the dopant, has been associated with photoelectron production giving rise to photon multiplication process when excess of energy is transferred to another electron via electron-electron collision. In the case of $\mathrm{Eu}^{3+}\left(4 f^{6}\right), \mathrm{Dy}^{3+}\left(4 f^{9}\right)$ and $\mathrm{Er}^{3+}\left(4 f^{11}\right)$, this absorption transition certainly overlaps $4 f-6 s$ parity allowed inter-configuration transitions which are expected very strong. The last ones appear clearly on the excitation spectra of $\mathrm{Tb}^{3+}$ doped into $\mathrm{LaCl}_{3}$ at approximately $117 \mathrm{~nm}(10.6 \mathrm{eV})$. This is a rather low energy process which can be explained if we consider that the ground configuration of $\mathrm{Tb}^{3+}\left(4 f^{8}\right)$ contains one electron more than the half-filled stable shell. This extra electron can be removed quite easily. 


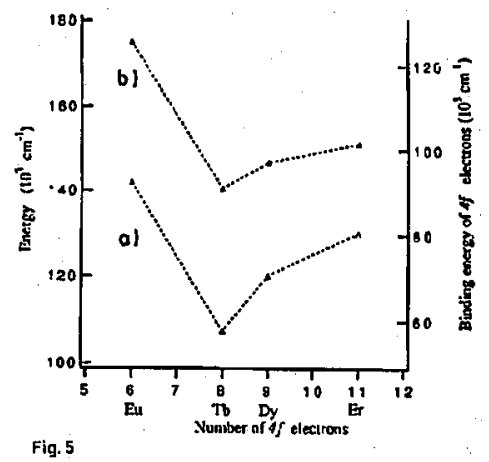

Fig. 5

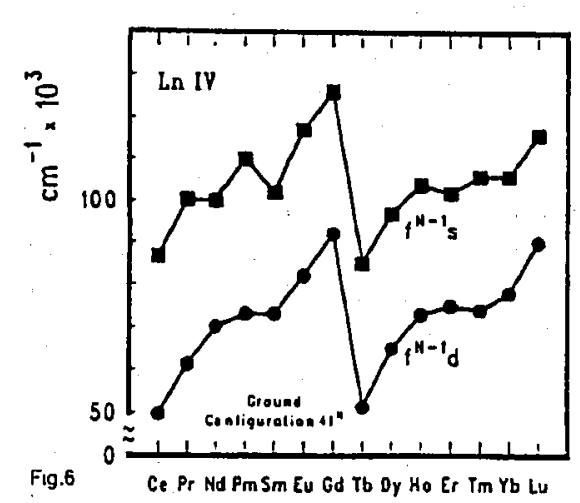
(from [3]). (b) Variation of the maximum of the highest energy recorded peak versus number of $f$ electrons (lines are drawn between the points just for eye convenience).

Fig. 6. Energies of electronic configurations of the triply ionised lanthanides (from [4]) .

At room temperature, the transitions between the ground state and the first excited configuration $4 f^{n-1} 5 d$ which are also parity allowed, cause broad bands widely vibronic in character. They show generally some structures. The plot of the lowest transition energy versus the number of $f$ electrons also follows the well-known energy variation calculated by Brewer [4] for inter-configuration transitions occurring in the tripositive lanthanide free ions (Fig. 6). In the condensed matter, the strong crystal field interaction due to the large radial extension of the $d$ orbitals shifts this transition energy toward lower energies and the importance of the shift is directly correlated to the crystal field magnitude. A special attention should be devoted to $\mathrm{Eu}^{3+}\left(4 f^{6}\right)$ with one $f$ electron less than the half-filled shell, which places the $4 f-5 d$ transitions relatively high in energy in agreement with the standard redox potential $E^{0}(3 / 4)$ value. More generally, the $5 d$ bandwidth arises from covalency and it is increased by low electronegativity ligands. For many lanthanide ions, the $4 f^{n-1} 5 d$ levels overlap widely those of the $4 f^{n}$ ground configuration in the UV and VUV energy region. For this reason, nonradiative relaxation can occur at room temperature between $5 d$ and $4 f$ levels.

When an electron is promoted from the ground state to $4 f$ excited levels, the $4 f \rightarrow 4 f$ resulting intra-configuration transitions give rise to weak and sharp peaks. They are generally electric dipolar transitions which are forbidden by the parity selection rules and forced by the crystal field configuration mixing. As a consequence, they are quite easy to assign.

\subsection{Charge transfer}

The observed structures on the excitation spectra described above are due to transitions in the lanthanide center itself between atomic-like states. Another important absorption mechanism is due to the transfer of one electron from anion 
to cation which involves the rare earth ion vicinity. In the fluoride and oxygen systems, the charge transfer occurs by electron delocalization from the filled $2 p$ shell of the ligand to the partially filled $f$ shell of the central rare earth ion. The encrgy of this band depends strongly on the electronegativity of the ligand and the electron affinity of the lanthanide ion as well as the ligand-metal distance. For most of the trivalent rare earth ions, especially around the middle of the lanthanide series, charge transfer and $f \rightarrow d$ transitions are overlapping strongly and the structure of the band cannot be completely resolved. We were pleased to notice that in $\mathrm{LaF}_{3}: \mathrm{Ln}^{3+}, \mathrm{O}^{2-}$ systems $(\mathrm{Ln}=\mathrm{Eu}, \mathrm{Dy}, \mathrm{Er})$, the intensity of the luminescence resulting from high energy configuration excitation, vanishes at low temperature. In these special cases, the only intense features remaining on the excitation spectra are due to charge transfer processes between $\mathrm{O}^{2-}-$ and $\mathrm{Dy}^{3+}$, $\mathrm{Er}^{3+}, \mathrm{Eu}^{3+}$, and between $\mathrm{F}^{-}$and $\mathrm{Eu}^{3+}\left(\mathrm{F}^{-} \rightarrow \mathrm{Er}^{3+}, \mathrm{Dy}^{3+}\right.$ occurring at too high energy to be detected). This phenomenon can be easily understood if we consider that at low temperature, the excited $d$ levels relax directly to the ground state multiplet with emission of VUV or UV photons [5]. This particular behaviour emphasizes in these systems the role of the oxygen contamination and especially the role of the $\mathrm{O}^{2-} \rightarrow \mathrm{Ln}^{3+}$ charge transfer band in the visible luminescence mechanism, at room temperature.

\section{Conclusion}

Different mechanisms which can seriously overlap, arise from the photon interactions with the solid in the 5 to $25 \mathrm{eV}$ energy region. In the large band gap systems of $\mathrm{LaF}_{3}$ doped with different rare earth ions, the complex excitation spectra of the visible luminescence become easier to interpret when temperature dependent experiments are achieved. Then, all the one-center processes, absorption and emission occurring in the rare earth ion itself can be localized as well as the charge transfer between the ligand and the metallic central ion. Unfortunately, in these luminescent systems, the host absorption process, namely the band to band transition, is not clearly distinguishable from characteristic activator bands. This means that the yield of rare earth ion luminescence is not high enough when excitation is in the fundamental excitation of the host. Plans for future work include investigation of different host materials in order to obtain valuable insight into many aspects of host excitation.

\section{References}

[1] R. Seedorf, H.J. Eichler, H. Koch, Appl. Opt. 24, 1335 (1985).

[2] I. Gérard, Thése de l'Université de Paris XI, 1993.

[3] G.K. Wertheim, A. Rosencwaig, R.L. Cohen, H.J. Guggenheim, Phys. Rev. Lett. 27, 505 (1971).

[4] L. Brewer, J. Opt. Soc. Am. 61, 1666 (1971).

[5] K.H. Yang, J.A. Deluca, Phys. Rev. B 17, 4246 (1978). 